\title{
Interactive comment on "Consequences of permafrost degradation for Arctic infrastructure - bridging the model gap between regional and engineering scales" by Thomas Schneider von Deimling et al.
}

\section{Anonymous Referee \#1}

Received and published: 27 October 2020

I enjoyed reading this paper, in which the authors propose the use of process-based tiling models (PTMs) to study the decade-scale impact of infrastructure placement on permafrost stability. Through a case study at the Dalton Highway in northern Alaska, the authors demonstrate the feasibility of PTMs for bridging the spatial gap between computationally expensive, short timescale geotechnical models (GTMs) and coarseresolution land surface models (LSMs). The authors apply process-based tiles within Cryogrid-3, an approach which has been used previously to model permafrost dynamics in other environments where lateral fluxes of water and energy are important. 
However, the present application of PTMs to infrastructure is novel, and the authors propose that it could be used to conduct pan-Arctic risk assessments for threats to infrastructure under different climate change scenarios. In general, I thought that the paper is well written, and the authors present an effective case study of the PTM approach at their field site. However, two general concerns I have are that: 1) I would like the authors to elaborate more on the outline they propose in section 4 for how to expand simulations like those reported here to a pan-Arctic risk assessment, and 2) I would appreciate a bit more discussion of the permafrost physics in the simulations from their Dalton Highway case study. In addition, I have several more specific comments, listed below.

\section{General comments:}

1) I appreciate that the authors sketch out how to incorporate PTMs into pan-Arctic risk assessments in section 4 , but currently the proposed workflow is somewhat difficult to understand. I know that this section is speculative, but l'd like a bit more detail about the connection between LSMs and PTMs. The authors state that results from an initial set of PTMs "could ... be used by LSMs to define a tiling-based model-setup for describing infrastructure in a reduced but manageable manner." Does this mean that a pan-Arctic LSM simulation would be executed, incorporating a sub-grid tiling scheme that accounts for the location of infrastructure? And if so, how would this be more informative than simply using meteorological forcing data derived from ESMs in the initial set of PTMs?

2) I am also curious at what stage of the analysis it is most appropriate to assess uncertainty related to the site-specific manners in which infrastructure may accelerate permafrost thaw. The authors acknowledge that "a specific gravel road can deviate strongly from our assumed setting here." As they state, one source of variability is road geometry, and another source of variability is that gravel roads can cause thaw through a variety of mechanisms, such as low-albedo dust deposition on the tundra or impedance to surface drainage. Another important factor to consider is that, unlike

Printer-friendly version

Discussion paper
Interactive comment 
the case study presented here, the effects of these process are often asymmetrical, occurring preferentially on one side of the roadway (e.g., Raynolds et al., 2014; Abolt et al., 2017). It seems like uncertainty in each of these factors would have a big impact on the risk assessment. Is it best to account for it within PTMs, LSMs, or GTMs?

3) The authors state in the abstract that rates of permafrost thaw adjacent to the highway follow "a two-phase beahvior", and in section 3.2, they state that thaw rates sharply increase "once a critical warming of the ground has been reached." This is an important result and I think it would be worthwhile for the authors to elaborate more on when this threshold occurs. For example, does the abrupt increase in thaw coincide with events such as the formation of an open talik, or the onset of surface water pondingâĂTor does it lag several years behind? Also, does the system stabilize by the end of the simulations? This information would be informative to the risk assessment, and I also think it would be interesting in the context of other numerical simulations of thermokarst.

Specific comments:

1) 72-74: This sentence is vague. I assume the issues referred to are related to infrastructure stability, but please be more specific.

2) 149-151: Please re-write this sentence or break it into two shorter ones.

3) 167-168: Please be more specific. Are you referring to improvements in subsurface physics that would allow ice lens formation to be simulated?

4) 176-178: This sentence is difficult to understand. Please consider rewriting it.

5) 183-185: Please include a concise definition of Process-based tiling models toward the start of this paragraph, including how they differ from the virtual tiling methods mentioned in the previous paragraph.

Printer-friendly version

6) 199: Replace the phrase "pan-Arctic wide" with "at the pan-Arctic scale."

Discussion paper

7) 205: Replace the phrase "we chose this region as our target region for modeling" 
with something like "we chose this region for our case study."

8) 221: Even though it's obvious, please begin this paragraph with a simple topic sentence, such as "Although our model was designed to capture essential components of permafrost thaw, it is limited by several physical assumptions." Also, you might consider specifying either here or in section 2.2 that your simulations are set up such that the primary driver of thaw is enhanced snow accumulation adjacent to the highway, as opposed to dust deposition or the backing up of surface flow.

9) 222-224: Please be more specific about how the constant bulk density of snow affects the subsurface. I assume this means that thermal conductivity is constant as well?

10) 236: Please define the acronym SNAP, and specify the time range for the forcing data here.

11) 264-265: I suggest rewording this to say "results in markedly warmer winter temperatures."

12) 366: Consider deleting the phrase "once a critical level of ground warming has been reached," as the first half of the sentence already references the idea of a threshold. In this paragraph, consider elaborating on when this threshold is reached.

13) What is an "external water flux"? Is this analogous to precipitation? Or lateral flow? And does it occur throughout the spatial domain? How did you settle on the amount of $2 \mathrm{~mm}$ per day?

Papers cited:

Raynolds et al., 2014. Cumulative geoecological effects of 62 years of infrastructure and climate change in ice-rich permafrost landscapes, Prudhoe Bay Oilfield, Alaska. Global Change Biology, 20, 1211-1224.

Abolt et al., 2017. Numerical modeling of ice wedge polygon geomorphic transition. 
Permafrost and Periglacial Processes, 28, 347-355.

TCD

Interactive comment on The Cryosphere Discuss., https://doi.org/10.5194/tc-2020-192, 2020.

Interactive

comment 